In Press at Computers in Human Behavior

\title{
Virtual Reality Check: Statistical Power, Reported Results, and the Validity of Research on the Psychology of Virtual Reality and Immersive Environments
}

\author{
Madison K. Lanier \\ Virginia Tech \\ T. Franklin Waddell \\ University of Florida \\ Malte Elson \\ Ruhr University Bochum \\ Daniel J. Tamul \\ Virginia Tech \\ James D. Ivory \\ Virginia Tech \\ Andrew K. Przybylski \\ University of Oxford
}

\begin{abstract}
Virtual reality (VR) is a popular subject of scientific study across a variety of academic fields. In the present study we evaluate methodological trends in behavioral research on VR with respect to data collection practices, statistical reporting, and data availability. In line with this goal, we conducted a meta-scientific analysis of 61 articles encompassing a total of 1,122 statistical tests and highlight three emergent trends that inform our understanding of past and future studies focused on VR. Conclusions from analysis of the data include a high incidence of errors in statistical reporting, and a general lack of transparency with respect to the availability of study data. Transparency in data analysis, increased statistical power, and more careful reporting of statistical outcomes are suggested to heighten methodological rigor and improve reproducibility in the field of VR research.
\end{abstract}

Keywords: Virtual reality; meta-science; statistical power; questionable research practices; quantitative methodology 


\section{Introduction}

Virtual reality, defined as a digital technology that uses stereoscopic displays to create three-dimensional content in an immersive and interactive experience, can evoke telepresence, or the feeling of being "elsewhere," away from one's physical environment (Steuer, 1992). While the technological fidelity of VR continues to advance, the dominant narrative in education, business, and technology studies is that VR can change or even save the world (Stein, 2015). Practitioners and scholars alike have been awed by the platform's capabilities and affordances, and have argued the technology affords users new perspectives, experiences, and environments that were never thought possible before (Fox, Arena, \& Bailenson, 2009).

Expectations for the technology have compounded as equipment for VR has become more affordable and easy to use - a development that has expanded the pool of researchers from military and industrial researchers to scholars in the social sciences interested in its prospective psychological effects (Fox, Arena, \& Bailenson, 2009 Steuer, 1992). This work has highlighted a variety of possible psychological outcomes VR might evoke, many of which have inspired an interest in the use of VR for practical applications. Independent of the promises of VR to society, the utility of VR to address research questions in social science research is necessarily dependent on the quality of the underlying methodologies and theories employed to study its influence on users. Fields such as psychology and communication are beginning to evaluate and critique the dominant methodological practices used in the field including topics such as sample size, data availability, and selective reporting (Elson \& Przybylski, 2017 Simmons, Nelson, \& Simonsohn, 2011). Given the impacts that VR technologies might have on users and society as a whole, scholars should take these developments into consideration when evaluating them through a social science lens. Otherwise, scholars could risk inadvertently misleading the general public when attempting to apply their research findings beyond the original laboratory setting. In line with this goal, our aim in the present study is to adopt a meta-methods approach that evaluates past research on VR along the dimensions of statistical power, the reporting of significant findings, and the frequency that transparent access to data is provided.

\section{Defining Virtual Reality: Approaches and Challenges}

There are a variety of approaches to both conceptualizing and researching VR technology. On the industry side, the technology and entertainment non-profit Virtual Reality Society defines VR as a type of immersive and realistic emulation that "present $[\mathrm{s}]$ our senses with a computer generated virtual environment that we can explore in some fashion" (Virtual Reality Society, 2017a). First-generation VR researchers including Ivan Sutherland and Myron Krueger similarly defined VR through the use of technological equipment systems. Sutherland, who created the first head-mounted display to connect to a computer, focused

James D. Ivory, Department of Communication, Virginia Tech, 181 Turner St NW, (0311), Shanks Hall, Room 121, Blacksburg, Virginia 24061. Phone: 540/231-7136. Fax: 540/231-9817. E-Mail: jivory@vt.edu M.E. is supported by the Digital Society research program funded by the Ministry of Culture and Science of North Rhine-Westphalia, Germany. 
on the use of special equipment to create the illusion of three-dimensional images (Sutherland, 1968). Krueger focused on "artificial reality" as immersive spaces using projectors, goggles, and reality gloves (Steuer, 1992). The name itself has changed over the years, emerging as "simulation" after the first flight simulator and eventually as "virtual reality" when coined by the inventor Jaron Lanier in the 1980s. Currently, the label "virtual environment" is applied in some scientific circles (Biocca, Kim, \& Levy, 1995. Virtual Reality Society, 2017b).

\section{VR in the Social Sciences}

In the late 1980's and throughout the 1990's, research on VR emerged as a topic of study in the social sciences. Notable early scholars include Frank Biocca, a communication researcher who was one of the first to study VR as a medium (Biocca, 1992; Lanier \& Biocca, 1992), and Jack Loomis, who helped introduce VR and presence to psychology (Loomis, Blascovich, \& Beall, 1999). In the late 90's, Jim Blascovich established one of the first major research centers focused on studying immersive VR in the social sciences (Fox, Arena, \& Bailenson, 2009). Arguing that VR is an extension of human faculties that involves both mass communication and interpersonal interaction, these scholars framed the medium as well-suited for studying both new and pre-existing psychological phenomena in these disciplines (Fox, Arena, \& Bailenson, 2009). While the graphical fidelity of such applications has been improved over time, the defining attributes of VR have been stable such as a first person perspective, head-mounted tracking, and a setting based in a virtual, three-dimensional setting. As a result, these early studies remain valuable for their insights into the effects of virtual simulations, even as contemporary forms of VR become more sophisticated and mainstream.

In the intervening years, social science research on VR has grown as VR has become more accessible and affordable as computing power and platforms have advanced. The dovetailing literature from both communication and psychology has created an expansive body of knowledge including studies of telepresence and violence (Tamborini et al., 2004), anxiety and phobias (Garcia-Palacios, Hoffman, Carlin, Furness, \& Botella, 2002; Klinger et al., 2005) imitation and eating behavior (Fox, Bailenson, \& Binney, 2009), and empathy and prosocial behavior (Kalyanaraman, Penn, Ivory, \& Judge, 2010). The methodologies used are often empirical, quantitative, and experimental in controlled, lab-based settings.

As VR has flourished, so too have the scope of inferences we draw about the technologies and humans as an area of study in the social sciences, it is important to evaluate the study of VR as it evolves. How are VR studies being designed? How are the results analyzed? How are these results presented? Each of these issues is considered in turn below.

\section{Challenge 1: Publication Bias and the File-Drawer Problem}

It is common for exciting and sensationalized stories to make the headlines (Grabe, Zhou, \& Barnett, 2001, Molek-Kozakowska, 2017), surprising results are the most likely to get published in scholarly journals. While such results are usually counterintuitive, such 
as a claim that hot cocoa increases senior citizens' intelligence, they have the support of statistical significance (Sorond, Hurwitz, Salat, Greve, \& Fisher, 2013). By comparison, null results often go unpublished - tucked away in a researcher's archives under the label "nothing special" (Simonsohn, Nelson, \& Simmons, 2014a). This issue, sometimes referred to as the "file-drawer problem," is a form of publication bias, where common-sense and nulleffect literature is often pushed aside to make room for more colorful results in academic journals. Over time, mundane and or inconclusive research data build up and collect dust in file drawers. As a result, it has been argued that the published literature becomes more distorted and less representative of actual scientific and behavioral phenomena (Ioannidis, 2005: Simonsohn et al., 2014a). If rare or fluke findings are the most likely to be published, a body of literature can accumulate primarily consisting of "happy accidents." Publication bias can lead to serious problems for the ability of other scholars to replicate the work done by others. If findings cannot be replicated using sound methodological practices, then positive results are unlikely to represent true phenomena (Simmons, 2016) or be useful to external audiences in applied settings. Discovery of high-rates of non-replication has led to what's called a replication crisis, an issue of social science that has hit the field of psychology particularly hard. In 2015, the Open Science Collaboration, an international consortium of researchers under the lead of Brian Nosek and other collaborators at the Center for Open Science attempted to replicate 100 psychological experiments in what they called the "Reproducibility Project." Their findings indicated that while 97\% of the original 100 studies reported having statistically significant results, only $36 \%$ of the replications actually did (Open Science Collaboration, 2015). These findings, and high-profile scandals involving fraud and faulty analyses sent shockwaves through psychology and created somewhat of an identity crisis for the field (Gelman, 2016; Palus, 2015, Verfaellie \& McGwin, 2011).

While the replication crisis describes a phenomenon occurring in psychology broadly, it bears a cautionary tale for those who would study and tout the potential impact of VR. Any field that relies upon null hypothesis significance testing, and operates within a culture that favors statistically significant results (as is the case with VR work) is likely to foster practices that lead to publication bias, such as repeatedly attempting to obtain positive results to support their favored hypotheses (Greenwald, 1975) or believing that null results are less likely to be published (Coursol \& Wagner, 1986). Given that VR studies often uses theory from fields that are currently experiencing such methodological quandaries, it is perhaps even more plausible that VR may have similar issues given the field's reliance on common theoretical and methodological practices from social psychology. Therefore, it is essential we critically examine and evaluate the statistical findings that have come out of communication, as well as those that come forward in the future.

\section{Challenge 2: Questionable Research Practices (QRPs)}

When journals are predisposed to only publish research that is statistically significant, authors whose livelihood depends on publication success are pressured to produce the surprising and unexpected results that top tier journals require for acceptance. Out of desperation to achieve the gold standard of statistical significance, some researchers adopt questionable research practices in order to get the positive results they desire. 
Questionable Research Practices (QRPs) have been described as "the steroids of scientific competition" (John, Loewenstein, \& Prelec, 2012). They can occur at any stage of the research process across a spectrum of severity; they are distinct from fraud in that they are not outright proscribed, but can still significantly change the findings and reporting of research to the point that they distort the findings of published literature.

Many QRPs are innocent errors that occur at the data collection stage of research. Some of these decisions, like using leading questions or biased language, can even be made unknowingly due to lack of knowledge, experience, or rigor. QRPs can also come into play in the analysis stage of research, such as excluding cases that have extreme scores (outliers), respond in certain patterns, or even fail to meet an unreasonable and/or arbitrary time limit (John et al., 2012). QRPs occur at the reporting stage as well. In order to achieve the putative standard of statistical significance, some researchers round their data in order to get closer to, and hopefully under, the critical .05 p-value. This can happen mathematically, where $p=.056$ gets incorrectly rounded down instead of rounded up and reported as less than .05 (John et al., 2012), as well as rhetorically, where the researcher employs optimistic phrases such as "marginally significant" or "approaching significance."

Finally, one QRP worth mentioning is a lack of transparency regarding the availability of deidentified laboratory data and materials. Many researchers do not make their data publicly available in cases where it would be difficult or impossible to identify individual participants (Elson \& Przybylski, 2017). Taking this decision obstructs other researchers from evaluating and incrementally building on published data, analyses, and research designs. If materials such as questionnaires that were used are not shared, the academic community is not able to use such materials or improve previously used measures and methodologies. With the exception of preregistered reports (Elson \& Przybylski, 2017), sometimes the only way a researcher's methods can be scrutinized is with original data and materials. Obtaining data and materials, however, is often difficult, as illustrated by Wicherts, Borsboom, Kats, and Molenaar's (2006) attempt to reanalyze data from 141 empirical articles in APA journals. After several months and 400 emails, the researchers had only received 36 of 249 data sets from corresponding authors.

\section{Gauging the Challenges: QRPs in VR Research}

At present, VR research in the social sciences is predominated by quantitative investigations which are almost all quasi or true experimental designs. In line with this, our evaluation of VR research is focused on investigations using experimental methods in communication and psychology. Specifically, our goal is to appraise the extent to which VR research in these areas does or does not subject itself to QRPs. While there are many QRPs that could be evaluated, we will focus on four elements - statistical power, $p$-values, reporting errors, and data availability - that respectively represent four stages of research: data collection, analysis, reporting, and sharing. Although this is by no means a comprehensive examination of VR literature, it helps us better detect the presence or absence of QRPs, and at which stages they may or may not occur. Importantly, consideration of statistical report-

ing is a necessary prerequisite to further critical engagement with the published literature 
on VR specific topics such as motion sickness or novelty effects; put simply, the foundation of VR must be verified before scholars can move further into VR specific imperatives.

A crucial element that enables a researcher to confidently report his or her findings is the element of statistical power, the probability that a null hypothesis will be correctly rejected conditional on a specified alternative hypothesis, alpha, and sample size (Murphy, Myors, \& Wolach, 2009). Once these parameters have been specified, a power analysis can demonstrate the probability of finding that effect (assuming it truly exists). Alternatively, based on the number of observations, researchers may calculate the power levels to detect a range of potential effect sizes. Power increases as the sample size increases, with a typical, if arbitrary, standard for minimum adequate power being $80 \%$, or $\beta<.20$. The smaller the number of observations, the less likely the findings of a given study are to be reliable (Ioannidis, 2005). Given the relative expense of VR technology, and the time needed to calibrate the technologies for human participants in a lab setting, the present study seeks to evaluate whether communication and psychology VR studies are adequately powered to detect the effect sizes typical in the area and standardized benchmarks proposed by Cohen (1992).

RQ1: What level of statistical power is typical in $V R$ research related to communication and psychology?

Pressures of publication bias can lead to the adoption of QRPs in data collection and analysis, such as $p$-hacking, in order to obtain the much sought after $p$-value below .05 . When looking at reported $p$-values across studies, publication bias is often detected based on an uptick of $p$-values near the .05 mark (Simonsohn et al., 2014a, 2014b), because it is mathematically improbable for values to collect around an arbitrary value given the known distribution of $p$ under specified hypotheses. Researchers from several fields including orthopaedics (Gagnier \& Morgenstern, 2017), epidemiology (Greenland et al., 2016), and psychology (Sellke, Bayarri, \& Berger, 2001) have pointed to widespread misuse of $p$-values in their disciplines. The American Statistical Association has even issued a statement on how $p$-values should be used, in part, because of their widespread misuse (Wasserstein \& Lazar, 2016). These works comprehensively overview common (mis)uses and (mis)interpretations of $p$-values. Most relevant to our own purposes is the fraught treatment of $p<.05$ as a "gold standard" for rejecting the null hypothesis because it indicates only a 1 in 20 chance that data are the result of random error. In short, $p$-values cannot be used as a probability that null is true or false (Wasserstein \& Lazar, 2016), and p-values at or slightly below .05 should be taken only as "weak evidence" to reject the null hypothesis (see Wasserstein \& Lazar, 2016, p. 132). This is in part because when the alternative hypothesis is true (i.e. an effect is "real"), $p$-values below .01 are far more likely to occur than any value between .01 and .05 (Lakens, 2014; Sellke et al., 2001). Thus, an uptick in $p$-values just below .05 is concerning: Those values are sufficient to publish but should be rare when effects are real.

One possible remedy to the reliance on $p$-values as the kingmaker of quantitative research is to also include some indication of effect sizes, "a quantitative reflection of the magnitude of some phenomenon that is used for the purpose of addressing a question of interest" (Kelley \& Preacher, 2012, p. 137). This is not a novel idea and the APA endorsed it two decades ago (Wilkinson \& Task Force on Statistical Inference, American Psychological 
Association, Science Directorate, 1999) in part because doing so can assist researchers in determining the practical significance of their work, even in the light of non-significant findings (Kirk, 1996).

Moderate $p$-hacking can usually get a researcher to a significance of .05 , while more ambitious $p$-hacking to, say the $p<.01$ level, is often difficult, obvious, and unsuccessful (Simonsohn, Simmons, \& Nelson, 2015). Keeping this in mind, a distribution of reported $p$-values skewed towards this .05 value can reveal much about publication bias and the file drawer problem in a given set of studies. $P$-curve and other methods provide researchers a tool to evaluate these $p$-values and quantify the chance of whether $p$-hacking or other questionable practices concerning error control are at play within a paper or subfield. In line with this, we explore the issue of publication bias in the VR literature by tabulating $p$-values reported in communication and psychology VR studies.

RQ2: What is the pattern of reported p-values in $V R$ research related to communication and psychology?

QRPs can also occur in the reporting of research, often in the form of systematic statistical errors and reporting typos. While these mistakes can be made in error, an overwhelming presence of error, particularly errors that incorrectly report statistical significance where there is none, can indicate a systemic preference for significance over accuracy in publishing research. If errors are made time and time again, and if these errors are made in only one direction (that of publishability, such as towards a $p$-value less than .05), it indicates a review process that systematically rewards researchers for not checking the accuracy of their results. Statistical packages like statcheck (Epskamp \& Nuijten, 2015) can aid researchers in checking their work before publishing (Nuijten, Hartgerink, van Assen, Epskamp, \& Wicherts, 2016). These programs also make it easy for researchers to check other researchers' work, so the present study aims to utilize this program to assess the overall reporting accuracy of communication and psychology VR research.

RQ3: What is the prevalence of errors made in reporting in $V R$ research results?

Finally, in sharing scientific knowledge, it is important for scholars to share how their findings and calculations were determined. To that end, a summary of how frequently VR scholars make available (or at least link to) supplemental materials and open data sets in their research is necessary to evaluate, which we test in RQ4.

RQ4: How often do VR studies related to communication and psychology reference supplemental data and materials?

\section{Materials and Method}

This study used a meta-scientific analysis to describe and analyze the methods used in VR experiments in communication and psychology research. The goal was to determine the average statistical power, distribution of reported $p$-values, rate of statistical reporting errors, and availability of data in these experiments. Such methods have previously been used by scholars to survey a field's existing body of knowledge for reliability, accountability, 
and accuracy - important values for replicability and credibility of scientific research (Elson \& Przybylski, 2017; Nuijten et al., 2016, Schimmack, 2012; Wicherts et al., 2006).

\section{Unit of Analysis}

For the first three research questions, the unit of analysis is each test statistic reported in the articles that meet the sampling criteria for inclusion (see https://osf.io/2x9cj/). That is, the statistical tests of VR-related hypotheses were coded as individual cases and assigned unique rows in a test-level dataset. However, RQ4 (and partially RQ1 in terms of reporting a power analysis) dealt with the referencing of supplemental materials on the article level, making the unit of analysis each individual article. To keep the analysis separate, an articlelevel dataset was created in addition to the test-level dataset.

\section{Sampling Procedure}

An article search was performed with parameters adapted from the meta-analysis performed by Cummings and Bailenson (2016) to calculate the aggregate effect of VR on presence. The procedure used by Cummings and Bailenson (2016) was modified to fit the research questions and methodological focus of this paper. Consistent with past systematic reviews of the literature, the article search started with two relevant online databases: PsycNET and Communication and Mass Media Complete.

The keywords "virtual reality" and "psychology" and "media" were used to search both databases, with results filtered to only peer-reviewed journals. The keyword search returned 779 articles from APA PsycNET, which were exported to EndNote and screened for duplicates. The same keyword search returned 59 articles from Communication and Mass Media Complete, which were also exported to EndNote, producing a total of 740 articles from both databases combined. In sum, a total of 705 unique articles were collected. Although this corpus excluded articles that were not formatted in APA style, there are many similarities across journals regardless of formatting, therefore any conclusions drawn from a subset of journals that are APA formatted would at minimum provide useful information about research in these journals, but more broadly, are be likely to tell us something about the field as a whole as well.

To determine which of the 705 articles would be analyzed to answer RQ1-RQ4, the following criteria were employed. First, only published journal articles were included, as the purpose of this study is to evaluate the accuracy and reliability of the current established body of knowledge of these disciplines. Next, only articles available as PDF or HTML files that included statistical analyses reported in APA format were included, as the statistical package used and described later in this paper requires specific information. In order to ensure consistency with statistical aggregation, only articles that were empirical in nature, using an experimental study design with clear predictions or expectations, were included. Consistent with Steuer's (1992) idea of VR as a human experience, the articles were included only if there was a use or presence of VR technology, understood here as using an immersive head-mounted display and/or tracking system, and one or more dependent 
variables measured human perception, affect, behavior, cognition, or other related factors. Finally, articles were excluded if they were not topically relevant to $\mathrm{VR}^{1}$ and/or did not pursue the study of communication, psychology, or a related social science 2 This dataset of coded articles is available at https://osf.io/jucrq/.

In total, $N=61$ articles met the sampling criteria. While inferential statistics require formal analyses to determine adequate sample size, descriptive studies of a field are typically evaluated in reference to similar analyses. The present work has a return rate of $8.65 \%$, which is consistent with meta-analyses that have previously been conducted with this area. Cummings and Bailenson's (2016) keyword search regarding presence and immersive technology returned over 200 articles, and 83 of these met their inclusion criteria. Similarly, a meta-analysis by Page and Coxon (2016) looking at Virtual Reality Exposure Therapy (VRET) analyzed 71 articles, and a keyword search by Gregg and Tarrier (2007) to explore the used of VR for mental health returned 3,036 articles, 50 of which met their primary inclusion criteria.

Taking this together the present study included a total of $K=1,122$ statistical tests extracted from the 61 articles meeting the sampling criteria $3^{3}$ These tests were coded in a test-level dataset so that they could be analyzed separately from the article-level data collected for RQ4. It should be noted that this final sample included articles that spanned more than 20 years of academic research on VR, during which time the graphical fidelity and action possibilities of VR has advanced considerably. In the present study, the issues that are under consideration (such as publication bias, reporting errors, and materials) are trends that one might assume are consistent regardless of VR fidelity. In other words, our study speaks to the typical patterns of statistical reporting in VR that tell us something about the culture and common practices of the field. These issues have been discussed in other fields for more than sixty years, and are likely to have been an impact to early VR research just as much as it is an important topic for contemporary VR work. All data and materials underlying this report are available at https://osf.io/8dxue/.

\section{Coded Variables}

Test-level dataset. Test statistics $(K=1,122)$ of the $N=61$ included articles were collected and coded in a test-level dataset according to a codebook, available at https: //osf.io/mt376/. Each row in the dataset represents one statistical test. Each test was assigned a case number and coded for basic publication info (same as for the article-level dataset), in addition to total sample size, number of between-subjects conditions, number of within-subjects conditions, number of participants per cell, subjects design (between,

\footnotetext{
${ }^{1}$ Articles studying augmented reality (AR) were excluded and coded as "not topically relevant to VR." This is because, as articulated by Baus and Bouchard (2014), VR seeks to extract a person from the real world and immerse them in a virtual or realistic one. AR is conceptually very different as it uses virtual elements to build upon the real world.

${ }^{2}$ Physiological articles and tests were excluded. This is not likely to have skewed sample and cell sizes in a downward direction, because many of these studies were clinical with only a few participants (or only one).

${ }^{3}$ Some articles included both psychological and physiological tests. Since solely physiological articles were excluded from the general article sample, physiological tests were also excluded from the test sample.
} 
within, or mixed), hypothesis tested, design, results, effect size, and recomputed $p$-value. In studies that had uneven cell sizes, the number of participants per cell was averaged. Each test statistic that meets the $p$-curve syntax and inclusion requirements was coded as a key result, and the selection method for these key results was coded in the adjoining column. Finally, if the case was a $t$-test and reported as one- or two-tailed, it was coded as such. Notes and descriptions were recorded in the final column in narrative format.

Only tests that included test statistics (even incomplete ones) were included in the dataset. However, specific tests that were detailed but not reported with test statistics, such as those that were just reported as "not significant," were included. These instances were coded as "NR" in the results column, as they still provided value for sample and cell size. However, unhypothesized, exploratory, and/or post-hoc tests that did not report any test statistics were not included (even as "NR" cases) as it could not be assumed how many were conducted and in what manner they were tested. This dataset of coded statistical tests is available at https://osf.io/9xp8n/.

\section{Analysis Strategy}

Calculating average power. Each test in the sample $(K=1,122)$ was coded for total sample size, number of between-subjects conditions, number of within-subjects conditions, subjects design (between-subjects or within-subjects), and number of participants per cell, per the procedure employed in prior communication research (Elson \& Przybylski, 2017). Additionally, each article meeting the sampling criteria $(N=61)$ was coded as to whether a power analysis was reported. Then, the average and median sample and cell size were calculated and compared to Cohen's (1992) recommended sample sizes for small, medium, and large effects.

Distribution of $\boldsymbol{p}$-values. As mentioned previously, a disproportionate number of significant findings with $p$-values very close to the $p=.05$ level suggest potential problems with trends in analysis practices. A valid distribution of true-effect $p$-values should be skewed to the right, with the most values occurring towards the low $(p<.01)$ end of the curve. If the curve is skewed to the left and a large number of $p$-values cluster around .05 , it can be inferred that research decisions are being made to achieve the golden .05 threshold often deemed "necessary" for publication. An online statistical tool available at http://www.p-curve.com offers an easy approach to generate $p$-distribution visualizations. With this web application, users can input a batch of test statistics and automatically generate a visual $p$-curve display for their samples (Simonsohn et al., 2014a, 2014b). In order to determine whether social scientific VR research is examining true effects, the relevant test statistics of the present sample that meet the application's syntax and selection requirements were input into this statistical tool.

Rate of reporting errors. Statistical reporting errors are relatively common in the fields of psychology (Nuijten et al., 2016) and communication research (Elson \& Przybylski, 2017; Vermeulen et al., 2015). Therefore, it is worthwhile to explore the reporting error rate of specific topic areas, such as VR research, within these disciplines. Statcheck (Epskamp \& Nuijten, 2015), a recently developed statistical $R$ package, provides a useful 
way of finding and examining these errors. Statcheck has been widely used to detect reporting errors (Nuijten et al., 2016; Veldkamp, Nuijten, Dominguez-Alvarez, van Assen, \& Wicherts, 2014) by serving as a "spellchecker" for null hypothesis significance testing by automatically aggregating, analyzing, and re-computing the $p$-values of a given sample of articles based on their test statistics. This function was used in the present study, by running the HTML or PDF files for the screened articles $(N=61)$ through statcheck. As an added measure of verification and accuracy, the individual tests themselves $(K=1,122)$ were also run through statcheck. In both exercises, the program estimated a general reporting error rate and then categorize these errors as either "inconsistent" or "grossly inconsistent." Gross inconsistencies refer to cases where insignificant $p$-values are incorrectly reported as statistically significant, and vice versa. Regular inconsistencies refer to reporting errors that do not straddle the $p=.05$ significance threshold. Frequencies of inconsistencies and gross inconsistencies were analyzed and reported as descriptive statistics.

Availability of materials and data. The use of open science practices can be beneficial to academic disciplines, such as the practice of making datasets and supplemental materials publicly available. All of the $N=61$ articles meeting our sampling criteria were closely read and coded for hyperlinks or references in the document linking to online data sets and/or supplemental materials. The frequency of such references were analyzed and reported as a descriptive statistic.

\section{Results}

\section{Descriptive Statistics}

The screened articles $(N=61)$ ranged in publication year from 2002 to 2018 . In total, 29 journals were represented, with the Annual Review of CyberTherapy and Telemedicine being the most frequently represented with 14 articles, followed by Media Psychology ( $n$ $=8)$ and Frontiers in Psychology $(n=7)$. At the test level $(K=1,122)$, the majority of statistical tests came from Frontiers in Psychology $(k=167)$, followed by Media Psychology $(k=157)$ and CyberPsychology, Behavior, and Social Networking $(k=150)$.

\section{Sample Size and Estimated Power}

To answer RQ1, descriptive statistical tests were conducted to determine the mean and median total sample sizes used for all tests in the sample, as well as the mean and median number of participants per condition/cell.

Across all statistical tests $(K=1,122)$, the mean total sample size was $M=48.29$ participants $(S D=33.25)$. The lowest occurring value was 4 total participants, and the highest occurring value was 182 participants. The median total sample size was 45 participants. The mean number of participants per condition (cell) was $M=33.65(S D=23.12)$. The lowest occurring value was two participants per condition, and the highest occurring value was 182 per condition. The median number of participants per condition was $M D=$ 25 (see Figure 1 for distribution). 


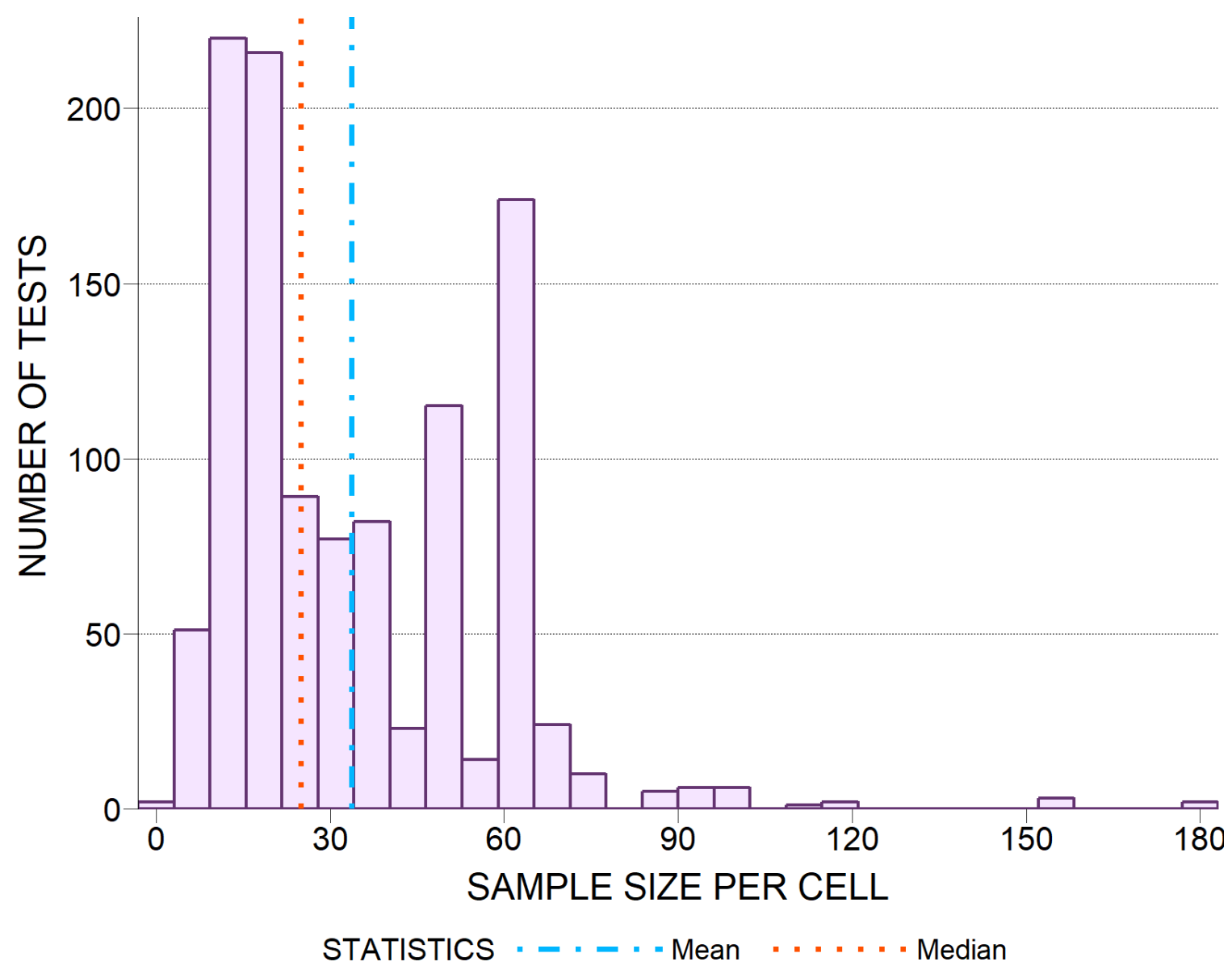

Figure 1. Sample size per condition across all statistical tests.

To estimate power levels, these numbers were compared to Cohen's (1992) recommended $n$ per cell to detect small, medium, and large effects in differences between two independent sample means at an alpha level of $\alpha=.05$. Compared to Cohen's (1992) recommended $n$ per group to detect large effects $(n=26)$, these numbers are adequate. Compared to the recommended $\mathrm{n}$ per group to detect medium effects $(n=64)$, these numbers are low. Compared the recommended $n$ per group to detect small effects $(n=393)$, these numbers are extremely low. Note that these comparisons are a general estimate, as they make broad generalizations about alpha level, statistical test, adequate power level (.80), and do not take into account other study design characteristics affecting statistical sensitivity (e.g., measurement precision).

To further evaluate RQ1, a planned article-level descriptive analysis was used to determine how many articles reported a power analysis. Of all the included articles $(N=$ $61)$, three articles $(4.92 \%)$ report a power analysis. Of these, two report a numerical power level and the effect size used. Only one article specifically mentioned conducting a power 
analysis a prior ${ }^{4}$ but none documented this as part of a preregistered sampling and analysis plan (Wagenmakers, Wetzels, Borsboom, van der Maas, \& Kievit, 2012).

\section{Distribution of $p$-values}

To address RQ2, the online application $p$-curve was used to generate a visual distribution of $p$-values reported in the sample. As discussed previously, inclusion rules were created based on recommendations by (Simonsohn et al., 2014a). Based on these rules, 160 tests were selected for $p$-curve analysis. Because $p$-curve only uses statistically significant results for its distribution, 55 nonsignificant tests at $p>.05$ were automatically excluded, resulting in 105 tests used for the final output. See Figure 2 for the observed $p$-curve, and Table 1 for its statistical description.

Visual analysis of the $p$-curve revealed that it was skewed to the right, with binomial $(p<.0001)$ and continuous $(p<.0001)$ tests indicating that the included tests contained evidential value. A small peak of values can be observed around $p=.03$. Of the included significant values, $76(72.38 \%)$ were $p<.025$. The $p$-curve estimates assume included studies are adequately powered, in this case at $85 \%$.

Table 1

Statistical description of the observed p-curve.

\begin{tabular}{|c|c|c|c|}
\hline & $\begin{array}{c}\text { Binomial Test } \\
\text { (Share of results } p< \\
.025 \text { ) }\end{array}$ & \multicolumn{2}{|c|}{$\begin{array}{c}\text { Continuous Test } \\
\text { (Aggregate with Stouffer Method) }\end{array}$} \\
\hline & & $\begin{array}{c}\text { Full } p \text {-curve } \\
(p \text { 's }<.05)\end{array}$ & $\begin{array}{l}\text { Half } p \text {-curve } \\
(p \text { 's }<0.25)\end{array}$ \\
\hline $\begin{array}{l}\text { 1) Studies containing } \\
\text { evidential value (Right Skew) }\end{array}$ & $p<.0001$ & $\begin{array}{c}Z=-16.21 \\
p<.0001\end{array}$ & $\begin{array}{c}Z=-16.9 \\
p<.0001\end{array}$ \\
\hline $\begin{array}{l}\text { 2) Studies' evidential value, if } \\
\text { any, is inadequate. (Flatter } \\
\text { than } 33 \% \text { power) }\end{array}$ & $p=.6889$ & $\begin{array}{l}Z=8.68 \\
p=.9999\end{array}$ & $\begin{aligned} Z & =18.27 \\
p & =.9999\end{aligned}$ \\
\hline $\begin{array}{l}\text { Power of tests included in } \\
p \text {-curve (correcting for selective } \\
\text { reporting) }\end{array}$ & \multicolumn{3}{|c|}{ Estimate: $85 \%, 90 \%$ Confidence interval: $(97 \%, 90 \%)$} \\
\hline
\end{tabular}

\section{Rate of Statistical Reporting Errors}

To answer RQ3, the PDF files for all the sampled articles $(N=61)$ were input into the $R$ statistical package statcheck. This program automatically culled all text-readable statistical tests in APA format and recomputed their true $p$-values. In total, statcheck

\footnotetext{
${ }^{4}$ When searching articles for the word "power," it was found that this term was most often used to refer to VR as a powerful medium.
} 


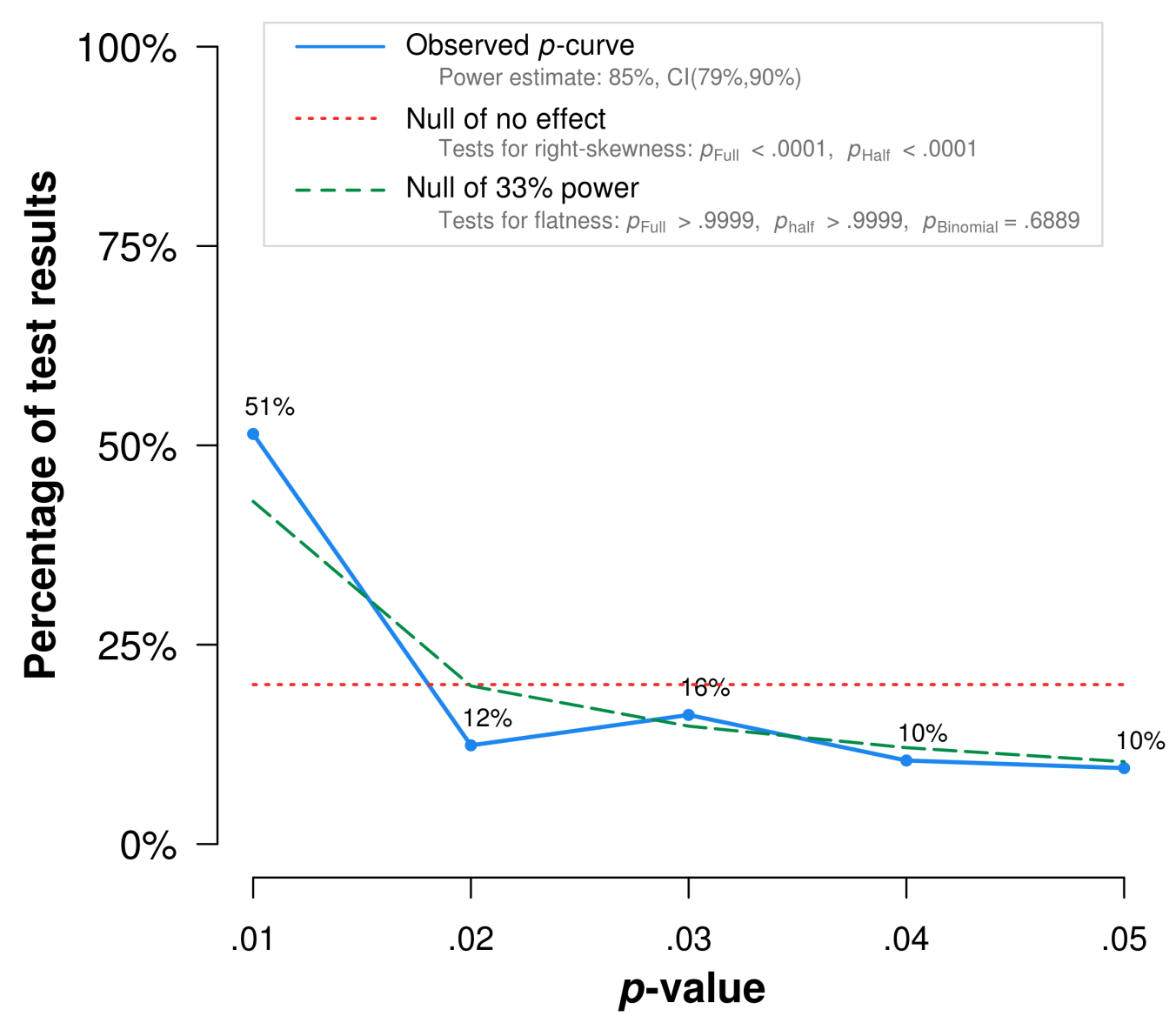

Note: The observed $p$-curve includes 105 statistically significant $(p<.05)$ results, of which 76 are $p<.025$. There were 55 additional results entered but excluded from $p$-curve because they were $p>.05$.

Figure 2. Observed $p$-curve.

found 198 significance tests among 23 of the files. Of these tests, 54 (27.27\%) were labeled "inconsistent," meaning that the test statistic, degrees of freedom, and $p$-value did not match. Of these, four tests (2.02\% of 198 total tests; $7.41 \%$ of 54 inconsistent tests) were labeled as "grossly inconsistent," meaning that the differences straddle the $\mathrm{p}<.05$ threshold of statistical significance ${ }^{5}$ In this case, these four tests were reported as significant $(p<$ $.05)$ even though reported test statistics and degrees of freedoms indicate they might not be $(p>.05)$.

\footnotetext{
${ }^{5}$ The actual statcheck output indicated 10 grossly inconsistent tests, however manually checking the article text revealed 6 tests that were labeled "grossly inconsistent" due to technical error with formatting (such as commas instead of decimals) or DF typos (switched numbers). The $p$-values for these 6 tests were technically reported correctly (albeit some with formatting errors) in their respective articles and were therefore excluded from the gross inconsistency count.
} 
Because of this low return rate $(17.65 \%)$, the raw test statistics from the dataset were input directly into statcheck's online application, www.statcheck.io. This produced a much higher return rate $(52.23 \%)$ with 586 tests returned through this method. Of these 586 tests, 89 (15.19\%) were labeled "inconsistent." Of these, 11 (1.88\% of 586 total tests; $12.36 \%$ of 89 inconsistent tests) were labeled "grossly inconsistent." A manual review of the statcheck output revealed all 11 of these tests to be reported as significant $(p<.05)$ even though reported test statistics and degrees of freedoms indicate they might not be $(p$ $>.05)$.

\section{Availability of Materials and Data}

To answer RQ4, the sampled articles were analyzed for references to supplemental data and materials. Across the articles $(\mathrm{N}=61)$, two (3.28\%) made references to supplemental data and materials. One was published in Frontiers in Human Neuroscience, and the other was published in Frontiers in Psychology. It is noteworthy that both were published in 2016 in journals which strongly encourage authors to share data and materials and include links in their papers so authors can access this information (Frontiers in Human Neuroscience, n.d.).

\section{Discussion}

In line with prior meta-scientific research on methodologies used in communication and psychology, this study found trends suggesting that there is room for improvement in methodological practice in the scientific pursuit of understanding VR's effects on users. Findings from this study speak to four main areas including statistical power, patterns of reported $p$-values, the frequency of reporting errors, and the availability of study materials.

Our first research question concerned the degree of statistical power or sensitivity typically provided in past VR research. In looking at VR experiments published in two well-known databases for psychology and communication research, it was found that the median cell size was 25 participants. Unless effects in VR research are relatively large (e.g.

a cell mean difference of $d>.8$ ), these studies may be underpowered (see Cohen, 1992). If average effect sizes are medium (e.g. a cell mean difference of $d=.5$ ), then on average there is only an $54 \%$ chance to detect them. At small effects of $d=.30$ or $d=.20$, researchers on average only have a $28 \%$ or $17 \%$ chance to detect them. Taken as a whole, past studies on the effects of VR have likely lacked adequate statistical power unless the effects under consideration were particularly large in magnitude, which is generally uncommon for studies in the social sciences.

Our second research question focused on the pattern of $p$-values that have been reported in past work on VR. Findings from a $p$-curve analysis based on aggregated $p$-values revealed a small peak of values around $p=.03$. The overall curve is indicative or some true, underlying effects; with some uncertainty, the small peak could be indicative of "data massaging" intended to produce statistically significant results through misuse of "researcher 
degrees of freedom" (Wicherts et al., 2016), such as multiple analyses and selective reporting of results. This type of undisclosed flexibility in data analysis can significantly increase the reporting of statistically significant false-positives (Simmons et al., 2011). For example, Simonsohn et al. (2015) found using data simulations that hacking to $p<.03$ requires about 25 alternative analyses and is successful about $45 \%$ of the time. Researchers and journal reviewers should therefore take extra caution in interpreting VR studies that report only significant findings or contain multiple $p$-values around the .01 to .05 level.

Our third research question concerned the frequency of possible errors in the reporting of statistical tests. An analysis of 586 statistical tests reported across 61 previously published papers revealed $15 \%$ of the reported tests included test statistics with inconsistent values, i.e. a mismatch between degrees of freedom, $p$-values, and the central test statistic for the analysis. In a minority of these instances, $p$-values were reported as statistically significant even though a manual inspection of the value revealed that the degrees of freedom and test statistics were consistent with $p$-values above the .05 threshold. Such a pattern of results suggests that there is much to be desired in terms of accurate reporting of $p$-values in published VR research. At the very least, this pattern of results should incentivize researchers to use methods like statcheck to assess the accuracy of their findings before submitting their work for peer review. At worst, a high rate of error in statistical reporting could bring into question the validity, accuracy, and usefulness of past studies on VR. In either case, editors and reviewers should be mindful of these possible shortcomings in reporting, either through their own careful inspection of reported results and/or by requesting authors to produce the findings of freely available data validation programs like statcheck.

Finally, our fourth research question shed light on how often, or not, past research on VR includes supplemental materials or access to study data. Our findings revealed that only two of 61 articles included reference to either supplemental materials or open data, thus suggesting that past work on VR has generally not followed best practices for open access and transparency. This finding should open the door for discussion about open science initiatives that might encourage future scholars to supplement submitted manuscripts with anonymized access to study materials. Such a practice would have multiple benefits including (1) fostering the capacity for outside replication, (2) archiving study materials to prevent loss of data or stimuli, and (3) enabling review of data and procedures by the broader academic community during the post-publication lifecycle of research.

\section{Methodological Recommendations for VR Research}

The results of the present study highlight a set of serious issues confronting the field of VR. Fortunately, these challenges are not insurmountable as there are clear paths forward which address these issues that scholars, editors, and the field as a whole should consider

moving to advance our empirical understanding of VR effects. First, we urge scholars who study the effects of VR to carefully consider the expected magnitude or smallest magnitude of interest of their VR intervention on key outcomes prior to beginning their study. Identifying the minimum effect size of practical and theoretical interest for a VR intervention allows researchers to determine the sample size that will be needed for their work to 
have adequate statistical power to detect effect sizes rooted in theoretical argument rather than opportunity. Second, we recommend increased multi-site collaboration to facilitate the completion of larger studies, particularly for VR studies that assume the effect of a possible intervention might be relatively subtle, such as the long-term attitudes or outcomes with multiple known predictors. Third, we suggest that scholars of VR increase the public availability of their VR simulations, questionnaires for measuring VR outcomes, and the data sets with accompanying code that informed their central conclusions. In short, while VR research requires unique considerations such as the availability of sophisticated equipment and a careful single subject protocol, we believe this work remains valuable and worthy of the extra effort necessary for these studies to be conducted. We hope the present manuscript provides an impetus for more collaboration across disciplines that will minimize the cost of work in this area while heightening the quality of evidence that such studies provide.

\section{Practical Implications}

This study underlines the importance of having critical discussions of academic integrity, which should affect the extent to which industries trust and invest in VR research. VR is a hot, and possibly overhyped, technology by today's standards, and as with any new innovation, industry leaders seek out expert recommendations in order to make decisions about its potential business value. They pass on the big promises that have been made to them, and it is critical to determine if VR can actually deliver. Furthermore, as with video games, computers, and TV predecessors before it, VR technology remains a new and mysterious media frontier. This study suggests that there continue to be pervasive unknowns and unanswered questions surrounding VR's effects and underscores the value social science research has to uncover its power as a medium for communication if we model methodological rigor in our research practices. Agencies like the Federal Communications Commission and Federal Trade Commission should be cautious when making decisions about VR technology until its effects are better understood and the research behind these effects is more methodologically sound.

\section{Limitations and Future Research}

This study contains several limitations that present countless opportunities for future research gauging the health of the research area. First, the present study provides a broad and general evaluation of the landscape of VR research in the social sciences. As such, we collapsed across diverse studies in terms of goals, methodologies, and findings to draw inferences about how VR is studied. The goal of this study was to look at this large body of research in the aggregate and evaluate its methodological trends, but the results presented here should not be used to draw definite conclusions about how VR technology effects its users. That said, study results would be useful as a guide for future research by suggesting potential areas of VR that may need a closer look as researchers shift to study Augmented, Mixed, and other developing so-called "XR" technologies. As this work progresses, one fruitful expansion of the present analysis would also be to quantify VR specific issues such as nausea in VR studies, the possibility for novelty effects, or issues related to the consistency 
of VR stimuli from subject to subject given the freedom that such interactive technologies afford to the user.

Second, like any meta-level analysis that aggregates previous research, is the sampling method used to select studies for analysis is necessarily bounded. While the two databases used, PsycNET and Communication and Mass Media Complete, have been similarly used in previous research (Cummings \& Bailenson, 2016, Hu, 2015), they may not represent the entire scope of social scientific VR research that exists today. Along similar lines, only journals with APA formatting were included, which was necessary to obtain test statistics that are less consistently reported in alternative formats. One consequence of these sampling choices is VR research conducted in military and industrial settings may not be listed in these databases, and therefore did not appear in the final sample. In the same vein, the keyword search used to find relevant articles was reliant on the phrase "virtual reality" to describe VR technology. As previously discussed, the constant shift in technology and naming conventions for this area of research makes it particularly difficult to label and capture. Today, terms like "mixed reality" and "virtual environments" are coming into the foray, while historically, terms like "stereoscopic" or "3D environments" could have also been used to describe the same phenomenon. Therefore, it is recommended that future research pursuing a historical overview of VR takes this into account when developing relevant search terms.

Third, on an article level, only studies that used immersive VR equipment with tracking technology (i.e., a HMD or headset) were included in order to narrow the technological scope. This was done to prevent the sample from becoming too broad, as introducing large bodies of research on video games and Human-Computer Interaction (HCI) could dilute the conceptual value of the current study. On the test-level, the present study only included psychological variables that were used in VR experiments. Physiological tests were excluded to prevent too much overlap with medical research on VR, as well as to help narrow the scope, but future researchers in this area should focus a meta-scientific lens consider including these tests as well.

Finally, the test selection criteria was not so narrow as to require the presence and absence of VR as an independent variable, and indeed, some of the included studies used multiple conditions that all took place in a VR environment. Again, lumping these tests together helps us get a big-picture look at the field, but it would be valuable to explore the specific study designs and manipulations being used in VR experiments.

Keeping these limitations in mind, we see the boundaries and limits described here as fruitful starting points for future research. The trends here point to quantifiable, observable shortcomings in VR research, but they are only scratching the surface. Fertile grounds for academic exploration include looking at what sampling methods have primarily been used, what relationships have been hypothesized, and possibly what qualitative research that has been done on VR. In fact, many articles that were not included in the final sample were editorials and journal commentaries that called for more VR research or touted VR as a powerful medium - but did not necessarily follow these claims with original data. It may be helpful for researchers and practitioners alike to see if the claims made in editorials matches (or possibly overshadows) the number of actual conclusive VR studies that have been done. 
Examining how these findings have been labelled, either as "exploratory" or "confirmatory," would be similarly useful. Finally, a replication of this study on newer XR would be useful as investment focus shifts into these areas.

\section{Conclusion}

The four research questions pursued in this study touch on four stages of the research process: Study design, data analysis, reporting findings, and sharing knowledge. The observations we report in this study suggest there are clear methodological challenges facing scientific practice at each of these four stages. This data provides an empirically-grounded cause for alarm for social scientists studying VR and a structure for further exploration. One can only hope that these findings can be used to better our understanding and pursuit of VR technology, and can propel us in a positive direction of sound research and scientific discovery. Robust, reproducible, and transparent social science research is the only path forward for VR research. 


\section{References}

Baus, O. \& Bouchard, S. (2014). Moving from virtual reality exposure-based therapy to augmented reality exposure-based therapy: A review. Frontiers in Human Neuroscience, 8. doi:10.3389/fnhum.2014.00112

Biocca, F. (1992). Communication within virtual reality: Creating a space for research. Journal of Communication, 42(4), 5-22. doi:10.1111/j.1460-2466.1992.tb00810.x

Biocca, F., Kim, T., \& Levy, M. R. (1995). The vision of virtual reality. In F. Biocca \& M. R. Levy (Eds.), Communication in the age of virtual reality (pp. 3-14). Hillsdale, NJ: Lawrence Erlbaum Associates.

Cohen, J. (1992). A power primer. Psychological Bulletin, 112(1), 155-159. doi:10.1037/ 0033-2909.112.1.155

Coursol, A. \& Wagner, E. E. (1986). Effect of positive findings on submission and acceptance rates: A note on meta-analysis bias. Professional Psychology: Research and Practice, 17(2), 136-137. doi:10.1037//0735-7028.17.2.136

Cummings, J. J. \& Bailenson, J. N. (2016). How immersive is enough? A meta-analysis of the effect of immersive technology on user presence. Media Psychology, 19(2), 272309. doi:10.1080/15213269.2015.1015740

Elson, M. \& Przybylski, A. K. (2017). The science of technology and human behavior: Standards, old and new. Journal of Media Psychology, 29(1), 1-7. doi 10.1027/1864$1105 / \mathrm{a} 000212$

Epskamp, S. \& Nuijten, M. B. (2015). statcheck: Extract statistics from articles and recompute p values. doi:http://CRAN.R-project.org/package=statcheck

Fox, J., Arena, D., \& Bailenson, J. N. (2009). Virtual reality: A survival guide for the social scientist. Journal of Media Psychology, 21(3), 95-113. doi:10.1027/1864-1105.21.3.95

Fox, J., Bailenson, J., \& Binney, J. (2009). Virtual experiences, physical behaviors: The effect of presence on imitation of an eating avatar. Presence: Teleoperators and Virtual Environments, 18(4), 294-303. doi:10.1162/pres.18.4.294

Frontiers in Human Neuroscience. (n.d.). Author Guidelines. Retrieved from https://www. frontiersin.org/journals/human-neuroscience\#author-guidelines

Gagnier, J. J. \& Morgenstern, H. (2017). Misconceptions, misuses, and misinterpretations of $\mathrm{p}$ values and significance testing. The Journal of Bone and Joint Surgery, 99(18), 1598-1603. doi:10.2106/JBJS.16.01314

Garcia-Palacios, A., Hoffman, H., Carlin, A., Furness, T., \& Botella, C. (2002). Virtual reality in the treatment of spider phobia: a controlled study. Behaviour Research and Therapy, 40(9), 983-993. doi:10.1016/S0005-7967(01)00068-7

Gelman, A. (2016, October 3). Why does the replication crisis seem worse in psychology? Slate. Retrieved from http://www.slate.com/articles/health_and_science/science/ 2016/10/why_the_replication_crisis_seems_worse_in_psychology.html

Grabe, M. E., Zhou, S., \& Barnett, B. (2001). Explicating sensationalism in television news: Content and the bells and whistles of form. Journal of Broadcasting 86 Electronic Media, 45(4), 635-655. doi:10.1207/s15506878jobem4504\{\_\}6

Greenland, S., Senn, S. J., Rothman, K. J., Carlin, J. B., Poole, C., Goodman, S. N., \& Altman, D. G. (2016). Statistical tests, p values, confidence intervals, and power: 
A guide to misinterpretations. European Journal of Epidemiology, 31(4), 337-350. doi:10.1007/s10654-016-0149-3

Greenwald, A. G. (1975). Consequences of prejudice against the null hypothesis. Psychological Bulletin, 82(1), 1-20. doi:10.1037/h0076157

Gregg, L. \& Tarrier, N. (2007). Virtual reality in mental health. Social Psychiatry and Psychiatric Epidemiology, 42(5), 343-354. doi:10.1007/s00127-007-0173-4

$\mathrm{Hu}$, Y. (2015). Health communication research in the digital age: A systematic review. Journal of Communication in Healthcare, 8(4), 260-288. doi:10.1080/17538068.2015. 1107308

Ioannidis, J. P. A. (2005). Why most published research findings are false. PLoS Medicine, 2(8), e124. doi:10.1371/journal.pmed.0020124

John, L. K., Loewenstein, G., \& Prelec, D. (2012). Measuring the prevalence of questionable research practices with incentives for truth telling. Psychological Science, 23(5), 524532. doi: $10.1177 / 0956797611430953$

Kalyanaraman, S., Penn, D. L., Ivory, J. D., \& Judge, A. (2010). The virtual doppelganger. The Journal of Nervous and Mental Disease, 198(6), 437-443. doi:10.1097/ NMD. 0b013e3181e07d66

Kelley, K. \& Preacher, K. J. (2012). On effect size. Psychological Methods, 17(2), 137-152. doi: $10.1037 / \mathrm{a} 0028086$

Kirk, R. E. (1996). Practical significance: A concept whose time has come. Educational and Psychological Measurement, 56(5), 746-759. doi:10.1177/0013164496056005002

Klinger, E., Bouchard, S., Légeron, P., Roy, S., Lauer, F., Chemin, I., \& Nugues, P. (2005). Virtual reality therapy versus cognitive behavior therapy for social phobia: A preliminary controlled study. CyberPsychology \& Behavior, 8(1), 76-88. doi:10.1089/cpb. 2005.8 .76

Lakens, D. (2014, May 29). The probability of p-values as a function of the statistical power of a test. Retrieved from http://daniellakens.blogspot.com/2014/05/the-probabilityof-p-values-as-function.html

Lanier, J. \& Biocca, F. (1992). An insider's view of the future of virtual reality. Journal of Communication, 42(4), 150-172. doi:10.1111/j.1460-2466.1992.tb00816.x

Loomis, J. M., Blascovich, J. J., \& Beall, A. C. (1999). Immersive virtual environment technology as a basic research tool in psychology. Behavior Research Methods, Instruments, \& Computers, 31(4), 557-564. doi:10.3758/BF03200735

Molek-Kozakowska, K. (2017). Stylistic analysis of headlines in science journalism: A case study of New Scientist. Public Understanding of Science, 26 (8), 894-907. doi:10.1177/ 0963662516637321

Murphy, K. R., Myors, B., \& Wolach, A. (2009). Statistical power analysis: A simple and general model for traditional and modern hypothesis tests. New York, NY: Routledge.

Nuijten, M. B., Hartgerink, C. H. J., van Assen, M. A. L. M., Epskamp, S., \& Wicherts, J. M. (2016). The prevalence of statistical reporting errors in psychology (1985-2013). Behavior Research Methods, 48(4), 1205-1226. doi:10.3758/s13428-015-0664-2

Open Science Collaboration. (2015). Estimating the reproducibility of psychological science. Science, 349(6251), aac4716. doi:10.1126/science.aac4716

Page, S. \& Coxon, M. (2016). Virtual reality exposure therapy for anxiety disorders: Small samples and no controls? Frontiers in Psychology, 7. doi:10.3389/fpsyg.2016.00326 
Palus, S. (2015, December 8). Diederik Stapel now has 58 retractions. Retraction Watch. Retrieved from http://retractionwatch.com/2015/12/08/diederik-stapel-now-has-58retractions/

Schimmack, U. (2012). The ironic effect of significant results on the credibility of multiplestudy articles. Psychological Methods, 17(4), 551-566. doi:10.1037/a0029487

Sellke, T., Bayarri, M. J., \& Berger, J. O. (2001). Calibration of p values for testing precise null hypotheses. The American Statistician, 55(1), 62-71. doi:10.1198/ 000313001300339950

Simmons, J. P. (2016, September 30). What I want our field to prioritize. DataColada. Retrieved from http://datacolada.org/53/

Simmons, J. P., Nelson, L. D., \& Simonsohn, U. (2011). False-positive psychology: Undisclosed flexibility in data collection and analysis allows presenting anything as significant. Psychological Science, 22(11), 1359-1366. doi:10.1177/0956797611417632

Simonsohn, U., Nelson, L. D., \& Simmons, J. P. (2014a). p-curve: A key to the file-drawer. Journal of Experimental Psychology: General, 143(2), 534-547. doi:10.1037/a0033242

Simonsohn, U., Nelson, L. D., \& Simmons, J. P. (2014b). p-curve and effect size: Correcting for publication bias using only significant results. Perspectives on Psychological Science, 9 (6), 666-681. doi $10.1177 / 1745691614553988$

Simonsohn, U., Simmons, J. P., \& Nelson, L. D. (2015). Better P-curves: Making P-curve analysis more robust to errors, fraud, and ambitious P-hacking, a Reply to Ulrich and Miller (2015). Journal of Experimental Psychology: General, 144(6), 1146-1152. doi: $10.1037 /$ xge0000104

Sorond, F. A., Hurwitz, S., Salat, D. H., Greve, D. N., \& Fisher, N. D. L. (2013). Neurovascular coupling, cerebral white matter integrity, and response to cocoa in older people. Neurology, 81(10), 904-909. doi:10.1212/WNL.0b013e3182a351aa

Stein, J. (2015, August 6). Why virtual reality is about to change the world. Time. Retrieved from http://time.com/3987022/why-virtual-reality-is-about-to-change-the-world/

Steuer, J. (1992). Defining virtual reality: Dimensions determining telepresence. Journal of Communication, 42(4), 73-93. doi:10.1111/j.1460-2466.1992.tb00812.x

Sutherland, I. E. (1968). A head-mounted three dimensional display. In Proceedings of the Fall Joint Computer Conference AFIPS, Part I (p. 757). New York, NY: ACM Press. doi:10.1145/1476589.1476686

Tamborini, R., Eastin, M. S., Skalski, P., Lachlan, K. A., Fediuk, T. A., \& Brady, R. (2004). Violent virtual video games and hostile thoughts. Journal of Broadcasting 8 Electronic Media, 48, 335-357.

Veldkamp, C. L. S., Nuijten, M. B., Dominguez-Alvarez, L., van Assen, M. A. L. M., \& Wicherts, J. M. (2014). Statistical reporting errors and collaboration on statistical analyses in psychological science. PLOS ONE, 9(12), e114876. doi $10.1371 /$ journal. pone.0114876

Verfaellie, M. \& McGwin, J. (2011, December). The case of Diederik Stapel. American Psychological Association. Retrieved from ttp://www.apa.org/science/about/psa/ 2011/12/diederik-stapel.aspx

Vermeulen, I., Beukeboom, C. J., Batenburg, A., Avramiea, A., Stoyanov, D., van de Velde, B., \& Oegema, D. (2015). Blinded by the light: How a focus on statistical "significance" may cause p-value misreporting and an excess of p-values just below .05 
in communication science. Communication Methods and Measures, 9(4), 253-279. doi: $10.1080 / 19312458.2015 .1096333$

Virtual Reality Society. (2017a). What is virtual reality? Retrieved from https://www.vrs. org.uk/virtual-reality/what-is-virtual-reality.html

Virtual Reality Society. (2017b). When was virtual reality invented? Retrieved from https: //www.vrs.org.uk/virtual-reality/invention.html

Wagenmakers, E.-J., Wetzels, R., Borsboom, D., van der Maas, H. L. J., \& Kievit, R. A. (2012). An agenda for purely confirmatory research. Perspectives on Psychological Science, 7(6), 632-638. doi $10.1177 / 1745691612463078$

Wasserstein, R. L. \& Lazar, N. A. (2016). The ASA's statement on p -values: Context, process, and purpose. The American Statistician, 70 (2), 129-133. doi:10.1080/00031305. 2016.1154108

Wicherts, J. M., Borsboom, D., Kats, J., \& Molenaar, D. (2006). The poor availability of psychological research data for reanalysis. American Psychologist, 61 (7), 726-728. doi:10.1037/0003-066X.61.7.726

Wicherts, J. M., Veldkamp, C. L. S., Augusteijn, H. E. M., Bakker, M., van Aert, R. C. M., \& van Assen, M. A. L. M. (2016). Degrees of freedom in planning, running, analyzing, and reporting psychological studies: A checklist to avoid p-hacking. Frontiers in Psychology, 7. doi:10.3389/fpsyg.2016.01832

Wilkinson, L. \& Task Force on Statistical Inference, American Psychological Association, Science Directorate. (1999). Statistical methods in psychology journals: Guidelines and explanations. American Psychologist, 54 (8), 594-604. doi:10.1037/0003-066X.54.8.594 